\title{
Effects of conjugated linoleic acid on growth performance, feed conversion efficiency, and subsequent carcass quality in broiler chickens
}

\author{
Beata Szymczyk ${ }^{1}$, Paweł M. Pisulewski ${ }^{2} *$, Witold Szczurek $^{1}$ and Piotr Hanczakowski ${ }^{1}$ \\ ${ }^{1}$ Department of Animal Nutrition, Institute of Animal Production, 32-083 Balice, Poland \\ ${ }^{2}$ Department of Human Nutrition, Agricultural University of Cracow, al. 29 Listopada 46, 31-425 Kraków, Poland
}

(Received 10 February 2000 - Revised 19 October 2000 - Accepted 31 October 2000)

\begin{abstract}
The effect of dietary conjugated linoleic acid isomers (CLA) on growth performance, carcass composition, fatty acid composition of adipose and muscle tissues, and serum lipoproteins was investigated in broiler chickens. A total of 160 (eighty male and eighty female) chickens were allocated to four dietary treatments $(0 \cdot 0,0.5,1 \cdot 0$, and $1.5 \%$ CLA) and fed a standard starter diet from 8 to $21 \mathrm{~d}$, and a grower-finisher diet from 22-42 d. When determined for the total period $8-42 \mathrm{~d}$, feed intake and body weight gains of broiler chickens were significantly reduced (from 3.31 to $3.12 \mathrm{~kg}$ and from 1615 to $1435 \mathrm{~g}$ respectively; $P<0.05$ ), particularly at the $1.5 \%$ dietary CLA level. Feed conversion efficiency and carcass yield values showed no significant effects of dietary CLA. Abdominal fat deposition was significantly reduced (from 2.68 to $1.78 \% ; P<0.05$ ), the relative proportion of breast muscles was unaffected, and that of leg muscles significantly increased (from 19.0 to $20.6 \% ; P<0.05$ ). The concentration of CLA isomers ( $\%$ of total methyl esters of fatty acids) increased linearly in tissue samples from broilers fed $0.5,1.0$, and $1.5 \%$ dietary CLA. The relative proportions of saturated fatty acids (16:0, 18:0) were significantly $(P<0 \cdot 01)$ increased, and those of monounsaturated $(16: 1,18: 1)$ and polyunsaturated fatty acids $(18: 2,20: 4$ in muscle tissues) significantly $(P<0.05)$ reduced. Total serum cholesterol concentrations reached a maximum in broilers fed $1.0 \%$ CLA and then decreased slightly (from 141.73 to $136.47 \mathrm{mg} / \mathrm{dl} ; P<0.01$ ). The same was true also for HDLcholesterol (from 113.58 to $109.97 \mathrm{mg} / \mathrm{dl} ; P<0.01$ ). The HDL cholesterol:total cholesterol ratio and serum triacylglycerol concentration was unaffected. In conclusion, feeding CLA to broiler chickens resulted in substantial incorporation of CLA isomers into their tissue lipids, thus providing a potential CLA-rich source for human consumption.
\end{abstract}

Conjugated linoleic acid: Growth performance: Fatty acid composition

Conjugated linoleic acid (CLA) is a mixture of positional and geometric isomers of linoleic acid (9cis,12cis octadecadienoic acid), containing a conjugated double bond system. The predominant CLA isomer is rumenic acid (9cis, 11trans octadecadienoic acid) which represents $90 \%$ of CLA present in milk and $75 \%$ of CLA present in beef fat (Chin et al. 1992). Rumenic acid was identified as an intermediate in the biohydrogenation of linoleic acid by the rumen bacterium Butyrivibrio fibrisolvens (Viviani, 1970). Consequently, ruminant products show relatively high concentrations of CLA $(0.5-1.5 \%$ of total fatty acids), whereas meats from monogastric animals are poor sources of these compounds $(0 \cdot 1-0 \cdot 2 \%$ of total fatty acids; Chin et al. 1992). The ability of humans to synthetize CLA from linoleic acid directly is equivocal (Herbel et al. 1998).
Instead, the bioconversion of trans-vaccenic acid to CLA in man was suggested (Santora et al. 2000).

Interest in CLA has increased in the past decade as a result of its potential beneficial health effects. Indeed, CLA was found to act as a growth factor (Chin et al. 1994), and a fat-to-lean repartitioning agent (Pariza et al. 1996; Park et al. 1997; Ostrowska et al. 1999), and to show anticarcinogenic (Schulz et al. 1992; Ip, 1997), hypocholesterolaemic and antiatherogenic (Lee et al. 1994; Nicolosi et al. 1997) properties. However, its role in the development of atherosclerosis is equivocal and needs further studies (Munday et al. 1999). CLA was also involved in stimulating the immune functions in chickens and rats (Cook et al. 1993). These immunostimulatory effects of CLA have been recently confirmed (Wong et al. 1998;

\footnotetext{
Abbreviations: CLA, conjugated linoleic acid; HDL-C, HDL-cholesterol; MUFA, monounsaturated fatty acids; PUFA, polyunsaturated fatty acids; TC, total cholesterol.

* Corresponding author: Professor Paweł M. Pisulewski, fax +48 12 6336245, email rrpisule@cyf-kr.edu.pl
} 
Hayek et al. 1999) in experiments using young and old mice. In humans, milk fat consumption as the major source of CLA (Jiang et al. 1999), was demonstrated to protect against the risk of breast cancer in women (Knekt et al. 1996). Also, CLA can inhibit the growth of human breast cancer cells (Visonneau et al. 1997) and prostate cancer cells (Cesano et al. 1998), when implanted into immunedeficient mice.

In view of the above health-related effects of CLA it seems desirable to provide CLA-enriched products for human consumption. It has been already demonstrated that CLA is readily incorporated in tissue lipids in mice (Belury \& Kempa-Steczko, 1997), rats (Chin et al. 1994; Sugano et al. 1997; Szymczyk et al. 2000), hamsters (de Deckere et al. 1999), and pigs (Kramer et al. 1998). The objective of the present study was to compare growth performance, feed conversion efficiency and carcass composition (fatty acid profiles) in broiler chickens fed commercial diets supplemented with either sunflower oil or graded levels of CLA. In addition, limited studies were conducted on serum lipoproteins in the chickens, in relation to intakes of CLA.

\section{Material and methods}

A total of $1601 \mathrm{~d}$ old (eighty male and eighty female) chickens of a commercial strain (Arbor Acres), obtained from a local hatchery, were housed in electrically heated battery brooders, and $24 \mathrm{~h}$ of light were provided. The birds were given access ad libitum to water and to the control starter diet, containing $4 \%$ of sunflower oil, for $7 \mathrm{~d}$ (Table 1 ). On day 8 , the chickens were randomly allocated to group

Table 1. Composition and nutrient content of standard experimental diets $(\mathrm{g} / \mathrm{kg})$

\begin{tabular}{lcc}
\hline \multirow{2}{*}{ Item } & \multicolumn{2}{c}{ Diets } \\
\cline { 2 - 3 } & Starter & Grower-finisher \\
\hline Ground maize & 477 & 500 \\
Ground wheat & 90 & 125 \\
Soybean meal & 350 & 290 \\
Sunflower oil & 40 & 40 \\
Limestone & 10 & 11 \\
Dicalcium phosphate & 20 & 21 \\
NaCl & 3 & 3 \\
Mineral and vitamin premixt & 10 & 10 \\
In 1 kg of dietł & & \\
Crude protein (g) & $215 \cdot 0$ & 193.6 \\
Met (g) & 4.7 & 4.0 \\
Lys (g) & 11.0 & 9.6 \\
ME (MJ) & $12 \cdot 6$ & 12.8 \\
Crude fibre (g) & 27.3 & 26.7 \\
Ca (g) & 9.3 & 9.6 \\
P avail. (g) & 4.2 & 4.2 \\
\hline
\end{tabular}

* The CLA product (60 \%) was substituted for sunflower oil $(0.83,1.66,2.5 \%)$ to obtain three experimental $(0.5,1.0,1.5 \% \mathrm{CLA})$ and control (0.0 CLA) groups.

† Premix provided per kg of diet: DKA-Starter: Vit. A, $8000 \mathrm{IU}$; Vit. $\mathrm{D}_{3}, 1200 \mathrm{IU}$; Vit. E, $10 \mathrm{mg}$; Vit. $\mathrm{K}_{3}, 2 \mathrm{mg}$; Vit. $B_{1}, 1.5 \mathrm{mg}$; Vit. $B_{2}, 4 \mathrm{mg}$; Vit. $B_{6}, 0.3 \mathrm{mg}$; Vit. $B_{12} 10 \mu \mathrm{g}$; D-calcium panthotenate, $8 \mathrm{mg}$; folic acid, $0.2 \mathrm{mg}$; nicotinamide, $12 \mathrm{mg}$; choline chloride, $150 \mathrm{mg}$; Mn, $50 \mathrm{mg}$; J, $0.3 \mathrm{mg}$; Zn $30 \mathrm{mg}$; Co, $0.4 \mathrm{mg}$; Se, $0.1 \mathrm{mg}$; DL-methionine, $1 \mathrm{~g}$. DKA-Grower/Finisher: Vit. A, 7000 IU; Vit. $D_{3}, 1000$ IU; Vit. E, 10 mg; Vit. K, 1.5 mg; Vit. $B_{2}, 4$ mg; Vit. $\mathrm{B}_{6}, 0.3 \mathrm{mg}$; Vit. $\mathrm{B}_{12} 10 \mu \mathrm{g}$, 10; D-calcium panthotenate, $3 \mathrm{mg}$; folic acid, $0.2 \mathrm{mg}$; nicotinamide, $10 \mathrm{mg}$; choline chloride, $200 \mathrm{mg}$; Mn, $50 \mathrm{mg}$; J, $0.2 \mathrm{mg}$; Zn, $30 \mathrm{mg}$; Co, $0.3 \mathrm{mg}$; Se, $0.2 \mathrm{mg}$; DL-methionine, $1 \mathrm{~g}$.

$\ddagger$ Calculated nutrient content.
Table 2. Fatty acid composition of the sunflower oil and the CLA supplement used in experimental diets (relative \%)

\begin{tabular}{|c|c|c|}
\hline Fatty acid & Sunflower oil & CLA \\
\hline $12: 0$ & - & 0.1 \\
\hline $14: 0$ & 0.01 & 0.1 \\
\hline $16: 0$ & $6 \cdot 2$ & 4.9 \\
\hline $16: 1$ & - & 0.1 \\
\hline 18:0 & - & $2 \cdot 0$ \\
\hline 9cis-18:1 & $24 \cdot 4$ & $29 \cdot 9$ \\
\hline 11 cis-18:1 & - & 0.7 \\
\hline $18: 2$ & $62 \cdot 9$ & - \\
\hline trans-18:2 & - & 0.8 \\
\hline 9 cis,11cis-18:2 & - & 0.5 \\
\hline CLA isomers (total): & - & $58 \cdot 7$ \\
\hline $9 c i s, 11$ trans-18:2 & - & 9.5 \\
\hline 8 trans,10cis-18:2 & - & $8 \cdot 6$ \\
\hline 11cis,13trans-18:2 & - & $9 \cdot 8$ \\
\hline 10trans,12cis-18:2 & - & $11 \cdot 2$ \\
\hline Other CLA & - & $19 \cdot 6$ \\
\hline $18: 3$ & 1.0 & - \\
\hline $20: 0$ & 0.3 & 0.9 \\
\hline $22: 0$ & 0.6 & 0.3 \\
\hline $24: 0$ & 0.2 & 0.1 \\
\hline Total fatty acids & $95 \cdot 6$ & $99 \cdot 1$ \\
\hline
\end{tabular}

cages. They were given a starter diet from 7 to $21 \mathrm{~d}$ and a grower-finisher diet from 22 to $42 \mathrm{~d}$ (Table 1). The four dietary treatments consisted of four graded levels $(0 \cdot 0,0 \cdot 83$, $1.66,2.5 \%$ ) of the commercial CLA product (Table 2) containing $60 \%$ CLA isomers (Natural Lipids Ltd., Hovdebygda, Norway). Thus, the resulting CLA dietary concentrations were $0 \cdot 0,0 \cdot 5,1 \cdot 0,1 \cdot 5 \%$ respectively. There were five replicates for each of four treatments, and each replicate cage contained eight broiler chickens. Body weights were recorded for each replicate on days 8,22 , and 42 , and feed intake was measured over these periods in order to calculate feed conversion efficiency.

At $42 \mathrm{~d}$ of age, eight birds (four male and four female) per each treatment, were stunned and slaughtered by neck cutting and exsanguinated. Blood samples were collected and serum samples were separated by low-speed centrifugation (1500 $\mathrm{g}$ for $15 \mathrm{~min})$ to determine lipoprotein profiles. Serum total cholesterol (TC) was analysed enzymatically with standard kits (Sigma-Aldrich, Poznan Poland), according to Allain et al. (1974) and its HDL fraction (HDL-C) according to Warrick et al. (1982). The LDL fraction of cholesterol was calculated as a difference between TC and HDL-C. Triacylglycerol content was estimated according to McGowan et al. (1983).

Carcasses were then plucked, and eviscerated to determine carcass weight, as a percentage of total weight, and abdominal fat (considered to be the fat extending within the ischium, surrounding the cloaca, and adjacent to the abdominal muscle) and breast and leg muscle weight as a percentage of carcass weight. Samples of abdominal fat, breast (pectoralis major and pectoralis minor) and leg (flexor cruris medialis) muscles were stored frozen $\left(-20^{\circ} \mathrm{C}\right)$ for further analysis.

The total tissue lipids (abdominal fat, breast and leg muscles) were extracted according to the method of Folch et al. (1957). They were saponified $\left(10 \mathrm{~min}, 75^{\circ} \mathrm{C}\right)$ in $0.5 \mathrm{M}$ $\mathrm{KOH}-$ methanol and then methylated $\left(10 \mathrm{~min}, 75^{\circ} \mathrm{C}\right)$ in $14 \% \mathrm{BF}_{3}-$ methanol (Morrison \& Smith, 1964). Finally, 
Table 3. Effect of dietary CLA level on growth performance and slaughter characteristics of chickens (Data are means for five replicates of each of four treatments for eight chickens per replicate)

\begin{tabular}{|c|c|c|c|c|c|c|c|c|c|c|c|c|c|}
\hline \multirow[b]{2}{*}{$\begin{array}{l}\text { Dietary level of } \\
\text { CLA (\%)† }\end{array}$} & \multicolumn{3}{|c|}{$\begin{array}{l}\text { Body weight gain } \\
\text { (g) in periods } \\
\text { (days) }\end{array}$} & \multicolumn{3}{|c|}{$\begin{array}{c}\text { Feed conversion } \\
\text { efficiency } \ddagger \\
\text { for periods (days) }\end{array}$} & \multicolumn{3}{|c|}{$\begin{array}{l}\text { Feed intake }(\mathrm{kg}) \\
\text { for periods } \\
\text { (days) }\end{array}$} & \multirow[b]{2}{*}{$\begin{array}{c}\text { Dressing } \\
\text { percentage }\end{array}$} & \multirow[b]{2}{*}{$\begin{array}{l}\text { Abdominal fat } \\
\text { deposition (\%) }\end{array}$} & \multicolumn{2}{|c|}{$\begin{array}{c}\text { Proportion (\%) to } \\
\text { mass of eviscerated } \\
\text { carcass }\end{array}$} \\
\hline & $8-21$ & $22-42$ & $8-42$ & $8-21$ & $22-42$ & $8-42$ & $8-21$ & $22-42$ & $8-42$ & & & $\begin{array}{c}\text { Breast } \\
\text { muscles }\end{array}$ & $\begin{array}{c}\text { Leg } \\
\text { muscles }\end{array}$ \\
\hline 0.0 & 468 & 1147 & $1615^{a}$ & 0.600 & 0.455 & 0.488 & $0.78^{\mathrm{A}}$ & 2.52 & $3 \cdot 31^{a}$ & 72.9 & $2 \cdot 68^{a}$ & 21.7 & $19 \cdot 0^{\mathrm{a}}$ \\
\hline 0.5 & 456 & 1112 & $1568^{\mathrm{a}}$ & 0.585 & 0.434 & 0.469 & $0.78^{\mathrm{A}}$ & 2.56 & $3 \cdot 34^{a}$ & 73.1 & $2.06^{\mathrm{ab}}$ & 20.9 & $20 \cdot 6^{\mathrm{ab}}$ \\
\hline 1.0 & 461 & 1073 & $1534^{\mathrm{ab}}$ & 0.599 & 0.424 & 0.465 & $0.77^{\mathrm{A}}$ & 2.53 & $3 \cdot 30^{\mathrm{a}}$ & 72.7 & $1.78^{\mathrm{b}}$ & 21.0 & $21 \cdot 3^{b}$ \\
\hline 1.5 & 434 & 1002 & $1435^{\mathrm{b}}$ & 0.629 & 0.412 & 0.460 & $0.69^{\mathrm{B}}$ & 2.43 & $3 \cdot 12^{b}$ & 71.6 & $1.94^{\mathrm{ab}}$ & $21 \cdot 1$ & $20 \cdot 4^{\mathrm{ab}}$ \\
\hline $\begin{array}{l}\text { SEM } \\
\text { Contrast§ }\end{array}$ & 6.08 & 19.81 & 20.86 & 0.008 & 0.006 & 0.005 & 0.010 & 0.024 & 0.032 & 0.262 & 0.131 & 0.245 & 0.329 \\
\hline Linear & NS & * & $\star \star \star \star$ & NS & * & * & $\star \star \star *$ & NS & * & NS & * & NS & NS \\
\hline Quadratic & NS & NS & NS & NS & NS & NS & * & NS & NS & NS & NS & NS & NS \\
\hline
\end{tabular}

$\dagger$ Mean values for CLA dietary level effect with different superscripts are significantly different at ${ }^{(\mathrm{a}, \mathrm{b})} P<0.05$ or ${ }^{(\mathrm{A}, \mathrm{B})} P<0.01$.

‡ Feed conversion efficiency is body weight gain/feed intake.

$\S$ Dietary CLA level effects were tested using linear and quadratic orthogonal contrasts. For details of procedures see $p .467$. ${ }^{\star} P<0.05$; ${ }^{\star \star} P<0.01 ;{ }^{\star \star \star} P<0.001$; NS $P \geq 0.05$.

fatty acid methyl esters were extracted with hexane and analysed on a Hewlett-Packard (model 5890) gas chromatograph, equipped with a BPX 70 fused silica capillary column (length $50 \mathrm{~m} \times 0.22 \mathrm{~mm}$ i.d. $\times 0.25 \mathrm{~mm}$ film thickness; SGE International, Ringwood, Victoria, Australia), and a flame ionisation detector. Helium was the carrier gas used at a split ratio of 50:1. The operating conditions were as follows: the temperature of injector was $210^{\circ} \mathrm{C}$, and that of detector was $240^{\circ} \mathrm{C}$. The initial oven temperature was $160^{\circ} \mathrm{C}$ for $35 \mathrm{~min}$, increasing progressively by $3^{\circ} \mathrm{C} / \mathrm{min}$ to $210^{\circ} \mathrm{C}$, and held constant at $210^{\circ} \mathrm{C}$ for $10 \mathrm{~min}$. The fatty acid percentage was integrated and calculated using the HP ChemStation computer program. Fatty acid methyl esters were identified by comparison of their retention times with authentic standards purchased from Sigma-Aldrich (Poland) and the CLA reference standards (9cis,11trans and 10trans, 12 cis isomers) were obtained from Larodan Fine Chemicals AB (Malmõ, Sweden). All analyses were performed at the Meat and Fat Research Institute (Warsaw, Poland).

The isomeric distribution of CLA in tissue lipids was expressed as a percentage of total CLA isomers present in the tissue total fatty acids:

$$
\begin{aligned}
& \text { CLA isomer profile }(\%) \\
& =\frac{\text { individual CLA isomer }(\% \text { of total FA) }}{\sum \text { CLA isomers }(\% \text { of total FA })} \times 100 .
\end{aligned}
$$

The data were analysed using either one-way (growth performance and slaughter characteristics) or two-way (tissue fatty acid composition and serum lipoproteins) ANOVA generated by the STATISTICA $v .5 \cdot 1$ package. In addition, dietary CLA level effects were tested using linear and quadratic orthogonal contrasts. Where appropriate, Duncan's multiple range test was used to determine the significance of differences between treatment means at the $P<0.05$ and $P<0.01$ levels of significance. The relationship between dietary CLA content and total CLA concentration in tissue lipids was also analysed by a linear regression analysis.

\section{Results}

Food intake of broiler chickens (Table 3), was significantly $(P<0.01)$ decreased (from 0.78 to $0.69 \mathrm{~kg}$ ) by dietary CLA over the starter $(8-21 \mathrm{~d})$ period, while no effects of CLA on this variable were noted over the grower-finisher (22-42 d) period. In spite of that, when determined for the total period 8-42 d, feed intake depression (from $3 \cdot 31$ to $3.12 \mathrm{~kg})$ was found to be statistically significant $(P<$ $0 \cdot 05)$. This response was also linear $(P<0 \cdot 05)$. Although body weight gains of birds (Table 3 ) were not affected significantly by dietary CLA over the starter (8-21 d) and the grower-finisher $(22-42 \mathrm{~d})$ period, they tended to decrease with increasing levels of CLA supplement. At the same time, when determined for the total period 8$42 \mathrm{~d}$, body weight gains of broilers were significantly $(P<$ $0.05)$ and linearly $(P<0.01)$ reduced (from 1615 to $1435 \mathrm{~g})$. There were no significant effects of dietary CLA on feed conversion efficiency (Table 3), particularly in younger birds $(8-21 \mathrm{~d})$. However, older birds (22-42 d) decreased (from 0.455 to 0.412 ) this measurement linearly $(P<0.05)$. The same was true for the total experimental period 8-42 d, during which, feed conversion efficiency decreased from 0.488 to 0.460 .

The carcass yield values, calculated on the basis of the carcass weight (after head and feet had been removed), and determined at the age of $42 \mathrm{~d}$, showed no significant effects of dietary CLA on the dressing percentage (Table 3). Abdominal fat deposition was significantly and linearly $(P<$ 0.05 ) reduced (from 2.68 for control to $1.94 \%$ for the group fed $2 \%$ dietary CLA respectively) whereas the relative proportion of breast and leg muscles (\% of carcass weight) responded differently to increasing levels of dietary CLA. The former variable was not affected by the treatment and the latter was significantly $(P<0 \cdot 05)$ increased.

Fatty acid composition of tissue lipids (abdominal fat, breast and leg muscles), expressed as a percentage of total 
Table 4. Effect of dietary CLA (\%) on relative (\%) fatty acid composition of abdominal fat in experimental chickens: cockerels ( $\left.0^{7}\right)$ and pullets ( $(9)$ (Data are means for five replicates of each of four treatments for eight chickens per replicate)

\begin{tabular}{|c|c|c|c|c|c|c|c|c|c|c|c|c|}
\hline & \multicolumn{4}{|c|}{ CLA dietary level (\%)† } & \multicolumn{2}{|c|}{ Contrast } & \multicolumn{2}{|c|}{$\operatorname{Sex}(S)$} & \multirow[b]{2}{*}{ SEM (pooled) } & \multicolumn{3}{|c|}{ Significance of effects } \\
\hline & 0.0 & 0.5 & $1 \cdot 0$ & 1.5 & $L$ & Q & $\sigma^{x}$ & q & & $\%$ & $\mathrm{~S}$ & $\% \times \mathrm{S}$ \\
\hline $14: 0$ & $0.37^{\mathrm{A}}$ & $0.54^{\mathrm{B}}$ & $0.71^{\mathrm{C}}$ & $0.77^{\mathrm{C}}$ & 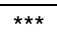 & NS & 0.58 & 0.62 & 0.030 & ** & NS & NS \\
\hline $16: 0$ & $17 \cdot 84^{\mathrm{A}}$ & $22 \cdot 17^{\mathrm{B}}$ & $26 \cdot 00^{C}$ & $26 \cdot 15^{\mathrm{C}}$ & NS & $* \star *$ & 22.78 & 23.28 & 0.667 & ** & NS & NS \\
\hline 7 cis- $16: 1$ & $0.34^{\mathrm{A}}$ & $0.21^{\mathrm{B}}$ & $0.27^{\mathrm{AB}}$ & $0.30^{\mathrm{A}}$ & $\star \star \star *$ & *** & 0.27 & 0.29 & 0.011 & $\star \star$ & NS & NS \\
\hline 9 cis-16:1 & $2 \cdot 47^{\mathrm{A}}$ & $1 \cdot 31^{\mathrm{B}}$ & $1.30^{\mathrm{B}}$ & $1.00^{\mathrm{B}}$ & $* \star *$ & $* *$ & 1.50 & 1.54 & 0.115 & ** & NS & NS \\
\hline $18: 0$ & $6 \cdot 10^{A}$ & $10 \cdot 74^{\mathrm{B}}$ & $13 \cdot 10^{C}$ & $13 \cdot 96^{\mathrm{C}}$ & $* \star *$ & *** & $10 \cdot 72$ & $11 \cdot 22$ & 0.566 & ** & NS & NS \\
\hline 9cis-18:1 & $33.09^{A}$ & $27 \cdot 20^{\mathrm{B}}$ & $26 \cdot 00^{\mathrm{B}}$ & $27 \cdot 10^{\mathrm{B}}$ & $* \star *$ & $\star \star * *$ & 28.54 & $28 \cdot 15$ & 0.537 & ** & NS & NS \\
\hline $11 \mathrm{cis}-18: 1$ & $1.36^{\mathrm{A}}$ & $0.90^{\mathrm{B}}$ & $0.85^{\mathrm{B}}$ & $1.05^{\mathrm{C}}$ & $\star \star *$ & $* * *$ & 1.03 & 1.05 & 0.039 & ** & NS & NS \\
\hline $18: 2$ & $36 \cdot 16^{A}$ & $32 \cdot 21^{B}$ & $23 \cdot 51^{C}$ & $17 \cdot 72^{\mathrm{D}}$ & $\star \star * *$ & NS & 27.55 & 27.29 & 1.350 & ** & NS & NS \\
\hline $18: 3 n-3$ & $0.76^{\mathrm{A}}$ & $0.66^{\mathrm{B}}$ & $0.60^{\mathrm{BC}}$ & $0.55^{\mathrm{C}}$ & $* \star *$ & NS & 0.64 & 0.65 & 0.018 & ** & NS & NS \\
\hline $18: 3 n-6$ & $0.20^{A}$ & $0.10^{B}$ & $0.07^{\mathrm{B}}$ & $0.01^{\mathrm{C}}$ & $\star \star * *$ & NS & 0.09 & 0.10 & 0.013 & ** & NS & NS \\
\hline CLA isomers & $0.00^{A}$ & $2 \cdot 94^{\mathrm{B}}$ & $6 \cdot 66^{C}$ & $10 \cdot 20^{\mathrm{D}}$ & $* \star *$ & NS & $5 \cdot 17$ & 4.73 & 0.700 & ** & NS & NS \\
\hline $20: 0$ & $0.11^{\mathrm{A}}$ & $0.17^{\mathrm{A}}$ & $0 \cdot 16^{\mathrm{A}}$ & $0.27^{\mathrm{B}}$ & *** & NS & $0 \cdot 18$ & 0.18 & 0.013 & ** & NS & NS \\
\hline $20: 1$ & $0.24^{A}$ & $0.27^{A B}$ & $0.29^{A B}$ & $0.34^{\mathrm{B}}$ & 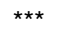 & NS & 0.27 & 0.29 & 0.010 & ** & NS & NS \\
\hline $20: 4$ & $0 \cdot 19^{A}$ & $0 \cdot 10^{\mathrm{B}}$ & $0.06^{\mathrm{BC}}$ & $0.01^{\mathrm{C}}$ & $\star \star * *$ & NS & 0.09 & 0.09 & 0.014 & ** & NS & NS \\
\hline Total SFA & $24.62^{\mathrm{A}}$ & $33.82^{\mathrm{B}}$ & $40 \cdot 40^{C}$ & $41 \cdot 36^{\mathrm{C}}$ & $\star \star \star *$ & $* \star *$ & 34.56 & 35.54 & 1.238 & ** & NS & $\star *$ \\
\hline Total MUFA & $37.60^{A}$ & $29 \cdot 90^{B}$ & $28.71^{B}$ & $29.57^{\mathrm{B}}$ & $\star \star \star *$ & $\star \star \star *$ & 31.65 & 31.35 & 0.679 & ** & NS & ** \\
\hline Total PUFA & $37.41^{\mathrm{A}}$ & $36 \cdot 24^{\mathrm{A}}$ & $31 \cdot 11^{\mathrm{B}}$ & $28.57^{\mathrm{B}}$ & $\star \star *$ & NS & 33.69 & 33.07 & 0.801 & ** & NS & $* *$ \\
\hline
\end{tabular}

SFA, saturated fatty acids; MUFA, monounsaturated fatty acids; PUFA, polyunsaturated fatty acids; L, linear; Q, quadratic; S, sex.

$\dagger$ Mean values for CLA dietary level effect with different superscripts are significantly different at $P<0.01$.

$\ddagger$ For details of statistical tests see p. 467. ${ }^{\star \star} P<0.01$; ${ }^{\star \star \star} P<0.001$; NS $P \geq 0.01$

methyl esters of fatty acids, was significantly altered by increasing dietary CLA concentrations (Tables 4, 5 and 6); the effect of sex was not detected. Generally, no CLA was found in the tissue lipids of broilers fed the control diet. In contrast, the concentration of CLA isomers was increased linearly $(P<0.001)$ in tissue samples (abdominal fat: $2 \cdot 94$, 6.66, and $10.20 \%$; breast: $2.89,5.25$, and $9.35 \%$; leg: $3 \cdot 22,5 \cdot 46$, and $10 \cdot 27 \%$ ) from broilers fed $0.5,1 \cdot 0$, and
$1.5 \%$ dietary CLA. No significant quadratic responses (except leg muscles) were detected. The relationship between dietary and tissue CLA concentrations are given in Fig. 1. Fatty acid profiles of tissue lipids did not reflect that of the commercial CLA product, thus indicating preferential incorporation of CLA isomers. Generally, the relative proportions of 9cis,11trans and 11cis,13trans CLA isomers in tissue lipids exceeded those found in the CLA

Table 5. Effect of dietary CLA (\%) on relative (\%) fatty acid composition of breast muscles in experimental chickens: cockerels ( $\left.\bigcirc^{\top}\right)$ and pullets $(\$)$ (Data are means of five replicates per treatment for eight chickens per replicate)

\begin{tabular}{|c|c|c|c|c|c|c|c|c|c|c|c|c|}
\hline & \multicolumn{4}{|c|}{ CLA dietary level (\%)† } & \multicolumn{2}{|c|}{ Contrast } & \multicolumn{2}{|c|}{$\operatorname{Sex}(S)$} & \multirow[b]{2}{*}{ SEM (pooled) } & \multicolumn{3}{|c|}{ Significance of effects } \\
\hline & 0.0 & 0.5 & $1 \cdot 0$ & 1.5 & $\mathrm{~L}$ & Q & $0^{x}$ & q & & $\%$ & $\mathrm{~S}$ & $\% \times \mathrm{S}$ \\
\hline $14: 0$ & $0.31^{\mathrm{A}}$ & $0.42^{\mathrm{AB}}$ & $0.51^{\mathrm{B}}$ & $0.55^{\mathrm{B}}$ & 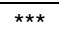 & NS & 0.43 & 0.47 & 0.022 & ** & NS & NS \\
\hline 16:0 & $19 \cdot 47^{\mathrm{A}}$ & $22 \cdot 21^{\mathrm{B}}$ & $24 \cdot 56^{C}$ & $24.59^{C}$ & $* * *$ & $\star *$ & $22 \cdot 21$ & $23 \cdot 21$ & 0.436 & $\star \star$ & NS & NS \\
\hline 7cis-16:1 & 0.30 & 0.22 & 0.24 & 0.22 & $\star *$ & NS & 0.25 & 0.24 & 0.010 & NS & NS & NS \\
\hline 9 cis $-16: 1$ & $1.76^{\mathrm{A}}$ & $1.01^{\mathrm{B}}$ & $0.97^{\mathrm{B}}$ & $0.75^{\mathrm{B}}$ & $* \star *$ & NS & $1 \cdot 14$ & $1 \cdot 11$ & 0.096 & $\star *$ & NS & NS \\
\hline $17: 0$ & 0.14 & 0.10 & 0.10 & 0.10 & $\star \star$ & NS & 0.11 & 0.11 & 0.005 & NS & NS & NS \\
\hline $18: 0$ & $8.87^{A}$ & $11 \cdot 77^{\mathrm{B}}$ & $13 \cdot 12^{\mathrm{BC}}$ & $13 \cdot 71^{C}$ & $* * *$ & $\star * *$ & $11 \cdot 71$ & $12 \cdot 03$ & 0.396 & $\star \star$ & NS & NS \\
\hline 9 cis-18:1 & $25 \cdot 00$ & 22.09 & $20 \cdot 05$ & $21 \cdot 10$ & NS & NS & 21.92 & $22 \cdot 20$ & 0.521 & NS & NS & NS \\
\hline 11 cis-18:1 & $1.95^{\mathrm{A}}$ & $1 \cdot 12^{\mathrm{B}}$ & $1.00^{\mathrm{B}}$ & $0.95^{\mathrm{B}}$ & $\star \star \star$ & NS & $1 \cdot 26$ & 1.25 & 0.077 & $\star \star$ & NS & NS \\
\hline $18: 2$ & $30 \cdot 70^{A}$ & $30 \cdot 11^{\mathrm{A}}$ & $26 \cdot 79^{A B}$ & $23 \cdot 17^{\mathrm{B}}$ & $\star \star \star *$ & NS & 28.43 & 26.95 & 0.729 & $\star \star$ & NS & NS \\
\hline $18: 3 n-3$ & 0.52 & 0.46 & 0.40 & 0.46 & NS & NS & 0.46 & 0.47 & 0.026 & NS & NS & NS \\
\hline $18: 3 n-6$ & $0.19^{A}$ & $0.14^{\mathrm{A}}$ & $0.04^{\mathrm{B}}$ & $0.01^{B}$ & $\star * \star$ & NS & 0.09 & 0.09 & 0.015 & $* \star$ & NS & NS \\
\hline CLA isomers & $0.00^{A}$ & $2 \cdot 89^{\mathrm{B}}$ & $5 \cdot 25^{\mathrm{C}}$ & $9 \cdot 35^{\mathrm{D}}$ & $\star \star \star *$ & NS & 4.46 & 4.29 & 0.647 & ** & NS & NS \\
\hline $20: 0$ & $0.07^{A}$ & $0.10^{\mathrm{AB}}$ & $0.11^{\mathrm{AB}}$ & $0.15^{\mathrm{B}}$ & $* \star *$ & NS & 0.11 & 0.11 & 0.008 & ** & NS & NS \\
\hline $20: 1$ & $0.26^{A}$ & $0.26^{A}$ & $0.30^{\mathrm{AB}}$ & $0.39^{\mathrm{B}}$ & $\star \star * *$ & NS & 0.31 & 0.29 & 0.014 & ** & NS & NS \\
\hline $20: 2$ & $0.89^{A}$ & $0.47^{\mathrm{B}}$ & $0.40^{\mathrm{B}}$ & $0.25^{\mathrm{B}}$ & $\star \star \star$ & NS & 0.51 & 0.50 & 0.058 & $\star \star$ & NS & NS \\
\hline $20: 3$ & 0.75 & 0.56 & 0.76 & 0.59 & NS & NS & 0.69 & 0.64 & 0.052 & NS & NS & NS \\
\hline $20: 4$ & $5.55^{A}$ & $3.81^{A B}$ & $3 \cdot 25^{\mathrm{AB}}$ & $1.84^{\mathrm{B}}$ & $\star \star \star$ & NS & $3 \cdot 61$ & 3.62 & 0.416 & $\star \star$ & NS & NS \\
\hline $20: 5$ & $0.00^{A}$ & $0.00^{A}$ & $0.22^{\mathrm{B}}$ & $0.30^{\mathrm{B}}$ & $\star \star \star *$ & * & 0.14 & 0.12 & 0.025 & ** & NS & NS \\
\hline $22: 4$ & $1.65^{A}$ & $1.00^{\mathrm{AB}}$ & $0.66^{\mathrm{B}}$ & $0.27^{\mathrm{B}}$ & $\star \star \star *$ & NS & 0.86 & 0.93 & 0.128 & ** & NS & NS \\
\hline $22: 5$ & 0.35 & 0.27 & 0.34 & 0.20 & NS & NS & 0.31 & 0.27 & 0.028 & NS & NS & NS \\
\hline $22: 6$ & 0.46 & 0.30 & 0.24 & 0.21 & $\star *$ & NS & 0.30 & 0.31 & 0.034 & NS & NS & NS \\
\hline Total SFA & $28.97^{A}$ & $34 \cdot 71^{\mathrm{B}}$ & $38.51^{C}$ & $39 \cdot 20^{C}$ & $\star \star \star *$ & $\star *$ & 34.67 & 36.02 & 0.814 & $\star *$ & NS & $\star *$ \\
\hline Total MUFA & $29 \cdot 34$ & 34.72 & $22 \cdot 56$ & $23 \cdot 41$ & $\star *$ & NS & 29.92 & $25 \cdot 10$ & 2.555 & NS & NS & * \\
\hline Total PUFA & $41.06^{a}$ & $40 \cdot 02^{a b}$ & $38 \cdot 35^{\mathrm{ab}}$ & $36 \cdot 66^{b}$ & ** & NS & 39.84 & 38.20 & 0.645 & * & NS & * \\
\hline
\end{tabular}

SFA, saturated fatty acids; MUFA, monounsaturated fatty acids; PUFA, polyunsaturated fatty acids; L, linear; $Q$, quadratic; S, sex. $\dagger$ Mean values for CLA dietary level effect with different superscripts are significantly different at ${ }^{(A, B, C)} P<0.01$ or at ${ }^{(a, b)} P<0.05$. ‡ For details of statistical tests see p. 467 . ${ }^{*} P<0.05 ;{ }^{* *} P<0.01$; ${ }^{* *} P<0.001$; NS $P \geq 0.05$. 
Table 6. Effect of dietary CLA (\%) on relative (\%) fatty acid composition of leg muscles in experimental chickens: cockerels ( $\left.{ }^{7}\right)$ and pullets (ㅇ) (Data are means of five replicates per treatment for eight chickens per replicate)

\begin{tabular}{|c|c|c|c|c|c|c|c|c|c|c|c|c|}
\hline & \multicolumn{4}{|c|}{ CLA dietary level (\%)† } & \multicolumn{2}{|c|}{ Contrast } & \multicolumn{2}{|c|}{$\operatorname{Sex}(S)$} & \multirow[b]{2}{*}{ SEM (pooled) } & \multicolumn{3}{|c|}{ Significance of effects } \\
\hline & 0.0 & 0.5 & 1.0 & 1.5 & L & Q & $0^{x}$ & q & & $\%$ & $\mathrm{~S}$ & $\% \times \mathrm{S}$ \\
\hline 14:0 & $0.36^{\mathrm{A}}$ & $0.49^{\mathrm{B}}$ & $0.54^{\mathrm{BC}}$ & $0.64^{\mathrm{C}}$ & $\star \star \star *$ & NS & 0.48 & 0.53 & 0.022 & ** & NS & NS \\
\hline $16: 0$ & $18.35^{\mathrm{A}}$ & $21 \cdot 30^{\mathrm{B}}$ & $23.05^{\mathrm{BC}}$ & $23.92^{C}$ & $\star \star * *$ & NS & $21 \cdot 17$ & $22 \cdot 13$ & 0.461 & ** & NS & NS \\
\hline 7 cis- $16: 1$ & $0.35^{\mathrm{A}}$ & $0.26^{\mathrm{B}}$ & $0.25^{\mathrm{B}}$ & $0.27^{A B}$ & ** & $\star \star$ & 0.29 & 0.28 & 0.011 & ** & NS & NS \\
\hline 9 cis $-16: 1$ & $2.45^{\mathrm{A}}$ & $1 \cdot 27^{\mathrm{B}}$ & $1.06^{\mathrm{B}}$ & $0.84^{\mathrm{B}}$ & NS & $\star *$ & 1.48 & 1.33 & 0.135 & $\star *$ & NS & NS \\
\hline $17: 0$ & 0.14 & 0.17 & 0.15 & 0.14 & $\star \star *$ & NS & 0.13 & 0.17 & 0.009 & NS & NS & NS \\
\hline 18:0 & $8.65^{\mathrm{A}}$ & $11.45^{\mathrm{B}}$ & $14 \cdot 46^{\mathrm{C}}$ & $13 \cdot 89^{C}$ & $* * *$ & $\star *$ & 11.77 & 12.45 & 0.471 & ** & NS & NS \\
\hline $9 c i s-18: 1$ & $26.59^{A}$ & $22 \cdot 92^{\mathrm{B}}$ & $20.04^{C}$ & $21.87^{\mathrm{BC}}$ & $* * *$ & ** & 23.37 & 22.34 & 0.567 & ** & NS & $\star * *$ \\
\hline 11 cis-18:1 & $1.61^{\mathrm{A}}$ & $0.99^{\mathrm{B}}$ & $0.91^{\mathrm{B}}$ & $0.95^{\mathrm{B}}$ & $* * *$ & $\star \star *$ & $1 \cdot 10$ & 1.13 & 0.056 & ** & NS & NS \\
\hline $18: 2$ & $33.36^{\mathrm{A}}$ & $31 \cdot 34^{\mathrm{A}}$ & $27 \cdot 06^{\mathrm{B}}$ & $22 \cdot 26^{\mathrm{C}}$ & $* \star *$ & NS & 28.99 & 28.02 & 0.872 & ** & NS & NS \\
\hline $18: 3 n-3$ & $0.70^{\mathrm{A}}$ & $0.69^{A}$ & $0.52^{\mathrm{B}}$ & $0.55^{\mathrm{AB}}$ & 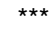 & NS & 0.62 & 0.61 & 0.022 & $\star \star$ & NS & NS \\
\hline $18: 3 n-6$ & $0.21^{\mathrm{A}}$ & $0.19^{A}$ & $0.05^{\mathrm{B}}$ & $0.01^{\mathrm{B}}$ & $* * *$ & NS & 0.11 & 0.12 & 0.017 & ** & NS & NS \\
\hline CLA isomers & $0.00^{A}$ & $3 \cdot 22^{\mathrm{B}}$ & $5 \cdot 46^{C}$ & $10 \cdot 27^{\mathrm{D}}$ & $* * *$ & ** & $4 \cdot 82$ & 4.66 & 0.686 & ** & NS & NS \\
\hline $20: 0$ & 0.11 & 0.12 & $0 \cdot 11$ & 0.12 & NS & NS & 0.12 & $0 \cdot 12$ & 0.007 & NS & NS & NS \\
\hline $20: 1$ & $0.25^{A}$ & $0.27^{\mathrm{A}}$ & $0.30^{\mathrm{A}}$ & $0.37^{\mathrm{B}}$ & $* * *$ & NS & 0.31 & 0.29 & 0.012 & ** & NS & NS \\
\hline $20: 2$ & $0.47^{\mathrm{A}}$ & $0.34^{B}$ & $0.30^{\mathrm{B}}$ & $0.16^{C}$ & *** & NS & 0.30 & 0.34 & 0.025 & $\star *$ & NS & NS \\
\hline $20: 3$ & 0.39 & 0.25 & 0.46 & 0.41 & NS & NS & 0.37 & 0.39 & 0.025 & NS & NS & NS \\
\hline $20: 4$ & $3 \cdot 87^{A}$ & $2 \cdot 60^{A B}$ & $3.26^{A B}$ & $1 \cdot 80^{\mathrm{B}}$ & * & NS & 2.64 & $3 \cdot 13$ & 0.261 & ** & NS & NS \\
\hline $20: 5$ & $0.00^{A}$ & $0.00^{\mathrm{A}}$ & $0.07^{\mathrm{B}}$ & $0.21^{C}$ & $\star \star \star \star$ & $\star \star \star *$ & 0.08 & 0.06 & 0.017 & ** & NS & NS \\
\hline $22: 4$ & $0.92^{\mathrm{A}}$ & $0.54^{\mathrm{B}}$ & $0.54^{\mathrm{B}}$ & $0.24^{B}$ & $* * *$ & NS & 0.50 & 0.62 & 0.062 & ** & NS & NS \\
\hline $22: 5$ & 0.22 & 0.16 & 0.25 & 0.20 & NS & NS & 0.19 & 0.22 & 0.016 & NS & NS & NS \\
\hline $22: 6$ & 0.26 & 0.20 & 0.20 & 0.16 & NS & NS & 0.18 & 0.23 & 0.019 & NS & NS & NS \\
\hline Total SFA & $27 \cdot 71^{\mathrm{A}}$ & $33.63^{\mathrm{B}}$ & $38.41^{\mathrm{C}}$ & $38 \cdot 81^{\mathrm{C}}$ & $* * *$ & $\star \star \star *$ & 33.78 & 35.50 & 0.873 & $\star *$ & * & $\star *$ \\
\hline Total MUFA & $31.60^{\mathrm{A}}$ & $25 \cdot 86^{\mathrm{B}}$ & $22 \cdot 67^{\mathrm{C}}$ & $24.41^{\mathrm{BC}}$ & $* * *$ & $\star * *$ & $26 \cdot 74$ & 25.53 & 0.742 & ** & NS & $\star \star$ \\
\hline Total PUFA & $40 \cdot 42^{\mathrm{a}}$ & $39.52^{\mathrm{a}}$ & $38 \cdot 18^{\mathrm{ab}}$ & $36 \cdot 29^{b}$ & ** & NS & 38.81 & 38.40 & 0.565 & * & NS & * \\
\hline
\end{tabular}

SFA, saturated fatty acids; MUFA, monounsaturated fatty acids; PUFA, polyunsaturated fatty acids; L, linear; Q; quadratic; S, sex.

$\dagger$ Mean values for CLA dietary level effect with different superscripts are significantly different at ${ }^{(A, B, C)} P<0.01$ or at ${ }^{(a, b)} P<0.05$.

¥ See text for more details of statistical tests. ${ }^{*} P<0.05 ;{ }^{* *} P<0.01$; ${ }^{\star \star *} P<0.001$; NS $P \geq 0.05$.

product. On the other hand, 8trans, 10cis and 10trans, $12 \mathrm{cis}$, and other CLA isomers were incorporated into tissue lipids less efficiently (Table 7). With regard to total fatty acid profiles, the relative proportions of saturated fatty acids were significantly $(P<0 \cdot 01)$ increased, due mainly to the increase in the concentration of palmitic (16:0) and stearic (18:0) acid. Saturated fatty acids responded also linearly $(P<0.001)$ and quadratically $(P<0.01)$. At the same time, the relative proportions of monounsaturated fatty acids (MUFA) in abdominal fat and leg muscles were significantly $(P<0.01)$ reduced. MUFA in these tissues responded also in a linear $(P<0.01)$ and quadratic $(P<$ $0.001)$ manner. Polyunsaturated fatty acids (PUFA) were significantly $(P<0.05)$ and linearly $(P<0.01)$ reduced. These latter changes resulted mainly from the fall in the concentration of palmitoleic (16:1), oleic (18:1), linoleic
(18:2) and arachidonic (20:4) acid (see: arachidonate in breast and leg muscles; Tables 5 and 6). Although present in negligible concentrations, the relative proportions of linolenic (18:3) acid were decreased (Tables 4, 5 and 6). In addition, concentrations of eicosapentaenoic (20:5) acid were increased and those of docosahexaenoic (22:6) decreased in breast and leg muscles (Tables 5 and 6) with increasing levels of dietary CLA. Generally, these responses were linear.

Serum lipoproteins were significantly affected by both treatments (dietary CLA and sex of birds; Table 8). TC concentrations reached a maximum $(141.73 \mathrm{mg} / \mathrm{dl})$ in broilers fed $1.0 \%$ CLA and then slightly decreased $(P<$ $0.01)$, resulting in a linear $(P<0.01)$ and quadratic $(P<$ $0 \cdot 05)$ effect. The same was true also for HDL-C responding in a quadratic $(P<0.05)$ manner. The resulting HDL-C:TC

Table 7. Comparison of CLA isomer profile (\%) of commercial CLA preparation, abdominal and muscle fat of experimental chickens

\begin{tabular}{|c|c|c|c|c|c|c|c|c|c|c|c|c|c|}
\hline & \multirow{3}{*}{$\begin{array}{c}\text { CLA } \\
\text { preparation }\end{array}$} & \multicolumn{4}{|c|}{ Abdominal fat } & \multicolumn{4}{|c|}{ Breast muscle } & \multicolumn{4}{|c|}{ Leg muscle } \\
\hline & & \multicolumn{4}{|c|}{ Dietary level of CLA (\%) } & \multicolumn{4}{|c|}{ Dietary level of CLA (\%) } & \multicolumn{4}{|c|}{ Dietary level of CLA (\%) } \\
\hline & & 0.0 & 0.5 & $1 \cdot 0$ & 1.5 & $0 \cdot 0$ & 0.5 & $1 \cdot 0$ & 1.5 & $0 \cdot 0$ & 0.5 & $1 \cdot 0$ & 1.5 \\
\hline \multicolumn{14}{|l|}{ CLA isomer: } \\
\hline 9 cis, 11 trans-18:2 & $16 \cdot 18$ & - & $19 \cdot 04$ & $20 \cdot 50$ & $20 \cdot 00$ & - & $17 \cdot 64$ & 18.85 & $19 \cdot 89$ & - & $18 \cdot 94$ & $19 \cdot 23$ & $20 \cdot 35$ \\
\hline 8 trans, 10 cis-18:2 & $14 \cdot 66$ & - & 11.90 & 9.90 & $10 \cdot 58$ & - & 8.65 & $6 \cdot 86$ & 8.56 & - & $10 \cdot 87$ & 7.51 & 8.47 \\
\hline 11 cis,13trans-18:2 & $16 \cdot 69$ & - & $23 \cdot 80$ & $23 \cdot 12$ & $22 \cdot 65$ & - & $29 \cdot 76$ & 35.52 & 28.56 & - & $27 \cdot 02$ & $30 \cdot 04$ & $26 \cdot 97$ \\
\hline 10trans,12cis-18:2 & $19 \cdot 08$ & - & $19 \cdot 04$ & $18 \cdot 62$ & $18 \cdot 72$ & - & $15 \cdot 22$ & $15 \cdot 24$ & $16 \cdot 26$ & - & 14.90 & 15.93 & $16 \cdot 85$ \\
\hline Other CLA & 33.39 & - & $25 \cdot 85$ & $27 \cdot 77$ & 27.94 & - & $25 \cdot 95$ & $25 \cdot 52$ & $25 \cdot 24$ & - & $29 \cdot 19$ & $27 \cdot 29$ & $27 \cdot 26$ \\
\hline
\end{tabular}

CLA isomer profile $(\%)=\frac{\text { individual CLA isomer }(\% \text { of total FA) }}{\sum \text { CLA isomers }(\% \text { of total FA })} \times 100$. 

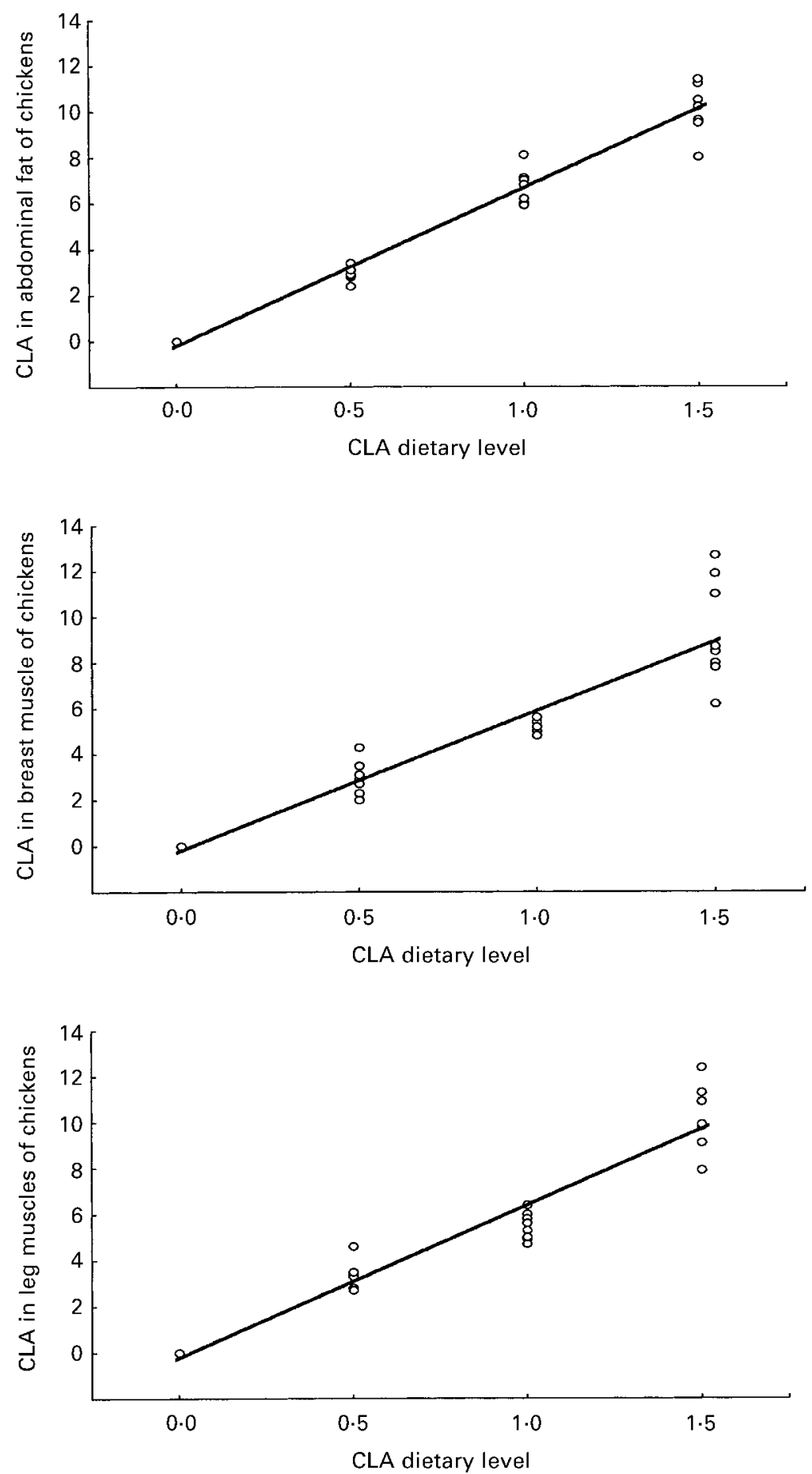

Fig. 1. Relationship between dietary CLA isomers content ( $g / 100 \mathrm{~g}$ diet) and proportions of CLA isomers ( $\mathrm{g} / 100 \mathrm{~g}$ total fatty acids) in abdominal fat (AF), breast (BM) and leg muscles (LM) of chickens. CLA dietary level $v$. CLA in AF, $r 0.98$ and $P=0.000000 ; v$. CLA in BM, $r 0.94$ and $P=0.000000 ; v$. CLA in LM, $r 0.97$ and $P=0.000000$.

tended to decrease (from 0.82 to 0.79 ). Although serum triacylglycerol concentration tended to increase (from 36.75 to $40 \cdot 14 \mathrm{mg} / \mathrm{dl}$ ), there was no significant effect of dietary CLA supplementation on this variable. Moreover, serum concentrations of TC and HDL-C were significantly $(P<0 \cdot 01)$ higher in males than in females.

\section{Discussion}

Depressed growth performance in broilers, resulting from feeding diets containing $0 \cdot 0,0.5,1 \cdot 0$, and particularly $1.5 \%$ dietary CLA, was consistent with earlier findings on mice (Belury \& Kempa-Steczko, 1997) and rats (Szymczyk 
Table 8. Effect of dietary CLA (\%) on serum lipoprotein in experimental chickens: cockerels $\left(\bigcirc^{\top}\right)$ and pullets $(Q)$

\begin{tabular}{|c|c|c|c|c|c|c|c|c|c|c|c|c|}
\hline & \multicolumn{4}{|c|}{ Dietary level of CLA (\%)† } & \multicolumn{2}{|c|}{ Contrastł } & \multicolumn{2}{|c|}{$\operatorname{Sex}(S)$} & \multirow[b]{2}{*}{ SEM (pooled) } & \multicolumn{3}{|c|}{ Significance of effects } \\
\hline & 0.0 & 0.5 & 1.0 & 1.5 & L & Q & $\sigma^{\pi}$ & ㅇ & & $\%$ & S & $\% \times S$ \\
\hline Total cholesterol (mg/dl) & $117 \cdot 39^{A}$ & $137 \cdot 41^{\mathrm{B}}$ & $141.73^{\mathrm{B}}$ & $136 \cdot 47^{\mathrm{B}}$ & ** & * & $144 \cdot 23$ & $122 \cdot 27$ & 1.12 & ** & *夫 & * \\
\hline HDL-cholesterol (mg/dl) & $96 \cdot 85^{\mathrm{A}}$ & $112.54^{\mathrm{B}}$ & $113.58^{\mathrm{B}}$ & $109 \cdot 97^{\mathrm{B}}$ & NS & * & $117 \cdot 16$ & $99 \cdot 31$ & 1.42 & $* *$ & ** & * \\
\hline HDL-cholesterol: & 0.82 & 0.82 & 0.79 & 0.80 & NS & NS & 0.81 & 0.81 & 0.007 & NS & NS & NS \\
\hline Triacylglycerols (mg/dl) & $36 \cdot 75$ & 34.73 & $35 \cdot 35$ & $40 \cdot 14$ & NS & NS & 38.89 & 34.59 & $1 \cdot 12$ & NS & NS & NS \\
\hline
\end{tabular}

$L$ linear; $\mathrm{Q}$, quadratic; $\mathrm{S}$, sex. ${ }^{*} P<0.05 ;{ }^{* \star} P<0.01 ; \mathrm{NS} P \geq 0.05$

$\dagger$ Mean values in the same row with different superscripts are significantly different at ${ }^{(\mathrm{A}, \mathrm{B})} P<0.01$.

‡ For details of statistical tests see p. 467.

et al. 2000), fed the $1.5 \%$ CLA-supplemented diet. In both studies marked reduction of growth variables (weight gains and feed conversion efficiency), relative to control animals, was observed. At the same time, our findings contrasted with those obtained earlier by Chin et al. (1994) in growing rats fed CLA. In the above work, the 0.25 and $0.50 \%$ CLA-supplemented diets enhanced feed intake, weight gains and feed conversion efficiency (in males and females) relative to control animals. Similarly, pigs fed diets containing $0.0-0.5 \%$ dietary CLA, tended to decrease feed intake and to increase weight gains, with concurrent significant improvements in feed conversion efficiency (Ostrowska et al. 1999). These data together suggest that dietary CLA has a potent effect on animal body mass resulting, most probably, from alterations in whole body metabolism. Thus, CLA was reported as a potent inhibitor of body fat accumulation in mice, rats, and chickens (Pariza et al. 1996). It was reported also to act as a fat-to-lean repartitioning agent in growing pigs (Dugan et al. 1997; Ostrowska et al. 1999). Consequently, these alterations in the ratio of fat-to-lean lead generally to improvements in feed conversion efficiency. However, at relatively high dietary CLA concentrations (exceeding $1.0 \%$ ), the beneficial changes in body composition do not bring expected improvements in growth performance. The possible explanation is that an excessive CLA treatment may stimulate fatty acid oxidation and thus enhance metabolic rate in animals, as demonstrated in mice (West et al. 1998).

The changes in broiler carcass composition were, at least partly, comparable with similar alterations reported earlier in pigs fed CLA-supplemented diets. Significantly reduced deposition of abdominal fat in broilers was in line with fatto-lean repartition, reduced back fat thickness and lower fat contents of commercial meat cuts in pigs fed CLA (Dugan et al. 1997; Dunshea et al. 1998; Thiel et al. 1998). In the same line, although the relative proportion (\% of carcass weight) of the breast muscles was not altered, that of the leg muscles was significantly $(P<0.05)$ decreased, confirming further favourable effects of CLA on animal carcass composition. These effects may be attributed to the ability of CLA to reduce body fat accretion with concurrent increases in lean tissue deposition as reported already in mice (Park et al. 1997) and more recently in pigs (Ostrowska et al. 1999). To offer an explanation, CLA has been found to reduce lipoprotein lipase activity and to increase lipolysis in cultured murine adipocytes (Park et al. 1997), thus leading to reduced fat deposition and favourably altered body composition.

As could be expected, feeding incremental levels of dietary CLA $(0.0-1.5 \%)$ resulted in linear increases in concentrations of CLA isomers in tissue lipids. Indeed, dietary CLA isomers were efficiently transferred in mice (Belury \& Kempa-Steczko, 1997), rats (Chin et al. 1994; Sugano et al. 1997; Szymczyk et al. 2000), hamsters (de Deckere et al. 1999), and pigs (Kramer et al. 1998), to various classes of body lipids. Moreover, incorporation of individual CLA isomers into body lipids differed as indicated by preferential incorporation of 9 cis,11trans and 11cis,13trans CLA at the expense of 8trans,10cis and 10trans,12cis, and other CLA isomers in our studies. Similarly, both 9cis,11trans and 10trans,12cis CLA isomers were incorporated into adipose tissue in rats (Sugano et al. 1997) and hamsters (de Deckere et al. 1999), but the former much more efficiently. Alternatively, there were no differences between the distribution of CLA isomers in the commercial CLA source (Natural Lipids Ltd.) fed to pigs, and in back fat, omental fat, liver and heart triacylglycerols (Kramer et al. 1998). Other striking results of our studies were the changes in the relative proportions of different classes of fatty acids in the abdominal fat, breast and leg muscles. Generally, the saturated fatty acid content of the above tissues was significantly $(P<0.01)$ increased and that of the MUFA and PUFA decreased $(P<0.05)$. These changes in the fatty acid profiles, were due mainly to increases in concentrations of 16:0 and 18:0, and concurrent opposite changes in concentrations of 16:1, 18:1, 18:2, and 20:4 (20:4 in breast and leg muscles). They could have resulted, at least partly, from the inhibition of $\Delta 9$ desaturase activity in the liver, caused by CLA, and impaired conversion of 18:0 to 18:1 (Lee et al. 1995, 1998). On the contrary, CLA increased 18:1 content in mouse liver lipids (Belury \& Kempa-Steczko, 1997) and hamster fat pads (de Deckere et al. 1999). The reduction in 18:2 and 20:4 concentrations is difficult to explain. However, it could be related to changes in 18:2, known to act as a metabolic precursor of 20:4. In fact, CLA gradually replaced sunflower oil (a rich source of linoleate) in the experimental diets. Correspondingly, dietary CLA decreased linoleate and arachidonate in mice (Belury \& Kempa-Steczko, 1997) and hamsters (de Deckere et al. 1999) liver lipids, rat adipose and muscle tissue (Szymczyk et al. 2000), and hamster fat pads (de Deckere et al. 1999). The reduction in arachidonate concentration has received particular attention as arachidonate-derived eicosanoids (prostaglandin $\mathrm{E}_{2}$, in particular) are frequently associated with the enhancement of cancer development (Belury \& Kempa-Steczko, 1997; Liu \& Belury, 1998). Finally, the changes in concentrations of 
18:3 and derived 20:5 (eicosapentaenoic acid) and 20:6 (docosahexaenoic acid) were equivocal. With apparent reduction in linolenate, the eicosapentaenoic acid concentrations were increased and those of docosahexaenoic acid decreased. We have no explanation for these effects.

Significant increases in serum TC concentrations, contradicted earlier results of different authors indicating hypocholesterolaemic effect of dietary CLA in different animal models (Lee et al. 1994; Nicolosi et al. 1997; Munday et al. 1999; Szymczyk et al. 2000). At the same time, the elevated concentrations of HDL-C were generally consistent with the above findings. However, the resulting ratio HDL-C:TC tended to decrease, indicative of an unfavourable lipoprotein profile (Griffin, 1999). Although insignificant, the increased concentrations of atherogenic triacylglycerols in our studies, confirmed previous findings of Szymczyk et al. (2000) on rats. Also, the CLA treatment resulted in elevated concentrations of serum non-esterified fatty acids in growing pigs (Ostrowska et al. 1999). This effect may result from inhibitory action of CLA on lipoprotein lipase coupled with stimulation of lipolysis in adipose tissue (Park et al. 1997). Consequently, reduced fat deposition and increased lipolysis in adipocytes could be responsible for elevated serum triacylglycerol concentrations, as found in broiler chickens. Interestingly, significant decreases in serum triacylglycerol concentrations in mice fed the $0.25 \%$ and $0.50 \%$ CLA-supplemented diets were reported by Munday et al. (1999).

In conclusion, our studies show that feeding CLA in incremental dietary concentrations $(0 \cdot 0-1.5 \%)$ to broilers is an effective way to obtain CLA-enriched poultry meat and thus the potential health-related benefits of CLA consumption in humans. At the same time, the broilers fed CLA-supplemented diets exhibit impaired growth performance. This negative effect is apparent particularly in broilers fed the $1.5 \%$ CLA-supplemented diet. Of the other effects, the deposition of abdominal fat is favourably reduced, the relative proportion of breast muscles (\% of carcass weight) is unaffected and that of leg muscles increased. The CLA supplement adversely affects the fatty acid composition of these tissues by increasing their saturated fatty acid content (e.g. 16:0, 18:0) at the expense of monounsaturated (e.g. 16:1, 18:1) and polyunsaturated (e.g. 18:2, 20:4) fatty acids. Further studies are certainly required to determine the optimum dietary concentration and balance of CLA isomers needed to obtain CLAenriched poultry meat and to avoid potentially adverse effects of CLA supplementation. In the context of our results it can be theoretically calculated that in order to provide the minimum required amount of CLA $(1.5 \mathrm{~g} / \mathrm{d}$; Decker, 1995), a $70 \mathrm{~kg}$ man would have to consume either a $1875 \mathrm{~g}$ portion of broiler breast or $300 \mathrm{~g}$ portion of broiler leg meat, obtained from birds fed the $1.5 \%$ CLAsupplemented diet. The same amount of CLA would be provided by $8.5 \mathrm{~kg}$ of $3.5 \%$ milk (Fritche \& Steinhart, 1998).

\section{Acknowledgements}

The authors wish to thank Professor Jan Wadstein (Natural Lipids Ltd., Hovdebygda, Norway) for providing the conjugated linoleic acid supplement and Professor $\mathrm{M}$. Obiedziński (Meat and Fat Research Institute, Warsaw, Poland) for analyses of tissue lipids. This work is supported by the State Committee for Scientific Research - Research Grant no 5-P06E-021-15.

\section{References}

Allain CC, Poon LS, Chan CS, Richmond W \& Fu PC (1974) Enzymatic determination of total serum cholesterol. Clinical Chemistry 20, 470-475.

Belury MA \& Kempa-Steczko A (1997) Conjugated linoleic acid modulates hepatic lipid composition in mice. Lipids 32, 199_ 204.

Cesano A, Visonneau S, Scimeca JA, Krichevsky D \& Santoli D (1998) Opposite effects of linoleic acid and conjugated linoleic acid on human prostatic cancer in SCID mice. Anticancer Research 18, 833-838.

Chin SE, Lin W, Storkson YL, Ha YL \& Pariza MW (1992) Dietary sources of conjugated dienoic isomers of linoleic acid, a newly recognized class of anticarcinogens. Journal of Food Composition Analysis 5, 185-197.

Chin SF, Storkson JM, Alblight KJ, Cook ME \& Pariza MW (1994) Conjugated linoleic acid is a growth factor for rats as shown by enhanced weight gain and improved feed efficiency. Journal of Nutrition 124, 2344-2349.

Cook ME, Miller CC, Park Y \& Pariza M (1993) Immune modulation by altered nutrient metabolism: nutritional control of immune-induced growth depression. Poultry Science 72, 1301-1305.

Decker EA (1995) The role of phenolics, conjugated linoleic acid, carnosine, and pyrroloquinoline quinone as nonessential dietary antioxidants. Nutrition Reviews 53, 49-58.

De Deckere EAM, van Amelsvoort JMM, McNeill GP \& Jones P (1999) Effects of conjugated linoleic acid (CLA) isomers on lipid levels and peroxisome proliferation in the hamster. British Journal of Nutrition 82, 309-317.

Dugan MER, Aalhus JL, Schaefer AL \& Kramer JKG (1997) The effect of conjugated linoleic acid on fat to lean repartitioning and feed conversion in pigs. Canadian Journal of Animal Science 77, 723-725.

Dunshea FR, Ostrowska E, Muralitharan M, Cross R, Bauman DL, Pariza MW \& Skarie C (1998) Dietary conjugated linoleic acid decreases back fat in finisher gilts. Journal of Animal Science 76, Suppl. 1, Journal of Dairy Science 81, Suppl. 1, 131.

Folch J, Lees M \& Sloane-Stanley GH (1957) A simple method for the isolation and purification of total lipids from animal tissues. Journal of Biological Chemistry 226, 497-509.

Fritche J \& Steinhart H (1998) Analysis, occurrence, and physiological properties of trans fatty acids (TFA) with particular emphasis on conjugated linoleic acid isomers (CLA) - a review. Fett/Lipid 100, 190-210.

Griffin BA (1999) Lipoprotein atherogenicity: an overview of current mechanisms. Proceedings of Nutrition Society 58, 163169.

Hayek MG, Han SN, Wu D, Watkins BA, Meydani M, Dorsey JL, Smith DE \& Meydani SN (1999) Dietary conjugated linoleic acid influences the immune response of young and old C57BL/ 6NCrlBR mice. Journal of Nutrition 129, 32-38.

Herbel BK, McGuire MK, McGuire MA \& Schulz TD (1998) Safflower oil consumption does not increase plasma conjugated linoleic acid concentrations in humans. American Journal of Clinical Nutrition 67, 332-337.

Ip C (1997) Review of the effects of trans fatty acids, oleic acid, $n$ 3 polyunsaturated fatty acids, and conjugated linoleic acid on 
mammary carcinogenesis in animals. American Journal of Clinical Nutrition 66, 1523S-1529S.

Jiang J, Wolk A \& Vessby B (1999) Relation between the intake of milk fat and the occurrence of conjugated linoleic acid in human adipose tissue. American Journal of Clinical Nutrition 70, 21-27.

Knekt P, Jarvinen R, Seppanen R, Pukkala E \& Aroma A (1996) Intake of dairy products and the risk of breast cancer. British Journal of Cancer 73, 687-691.

Kramer JK, Sehat N, Dugan ME, Mossagba MM, Jurawecz MP, Roach JA, Eulitz K, Aalhus JL, Schaefer AL \& Ku Y (1998) Distribution of conjugated linoleic acid (CLA) isomers in tissue lipid classes of pigs fed a commercial CLA mixture determined by gas chromatography and silver ion-high-performance liquid chromatography. Lipids 33, 549-558.

Lee KN, Kritchevsky D \& Pariza MW (1994) Conjugated linoleic acid and atherosclerosis in rabbits. Atherosclerosis 108, 19-25.

Lee KN, Storkson JM \& Pariza MW (1995) Dietary conjugated linoleic acid changes fatty acid composition in different tissues by decreasing monounsaturated fatty acids. 1995 IFT Annual Meeting/Book of Abstracts, 183.

Lee KN, Pariza MW \& Ntambi JM (1998) Conjugated linoleic acid decreases hepatic stearoyl-CoA desaturase mRNA expression. Biochemical and Biophysical Research. Communications 248, 817-821.

Liu KL \& Belury MA (1998) Conjugated linoleic acid reduces arachidonic acid content and $\operatorname{PGE}(2)$ synthesis in murine keratinocytes. Cancer Letters 127, 15-22.

McGowan MW, Artiss JD, Strandbergh DR \& Zak B (1983) A peroxidase-coupled method for the colorimetric determination of serum triglicerides. Clinical Chemistry 29, 538-542.

Morrison WR \& Smith LM (1964) Preparation of fatty acid methyl esters and dimethylacetals from lipids with boron fluoride-methanol. Journal of Lipid Research 5, 600-608.

Munday JS, Thompson KG \& James KAC (1999) Dietary conjugated linoleic acids promote fatty streak formation in the C57BL/6 mouse atherosclerosis model. British Journal of Nutrition 81, 251-255.

Nicolosi RJ, Rogers EJ, Kritchevsky D, Scimeca JA \& Huth PJ (1997) Dietary conjugated linoleic acid reduces plasma lipoproteins and early atherosclerosis in hypercholesterolemic hamsters. Artery 22, 266-277.

Ostrowska E, Muralitharan M, Cross RF, Bauman DE \& Dunshea FR (1999) Dietary conjugated linoleic acids increase lean tissue and decrease fat deposition in growing pigs. Journal of Nutrition 129, 2037-2042.
Pariza MW, Park Y, Cook M, Albright K \& Liu W (1996) Conjugated linoleic acid (CLA) reduces body fat. FASEB Journal 10, A560, (Abst.).

Park Y, Albright KJ, Liu W, Storkson JM, Cook ME \& Pariza M (1997) Effect of conjugated linoleic acid on body composition in mice. Lipids 32, 853-858.

Santora JE, Palmquist DL \& Roehrig KL (2000) Trans-vaccenic acid is desaturated to conjugated linoleic acid in mice. Journal of Nutrition 130, 208-215.

Schulz TD, Chew BP, Seaman WR \& Luedecke LO (1992) Inhibitory effect of conjugated dienoic derivatives of linoleic acid and beta-carotene on the in vitro growth of human cancer cells. Cancer Letters 63, 125-133.

Sugano M, Tsujita A, Yanmasaki M, Yamada K, Ikeda I \& Kritchevsky D (1997) Lymphatic recovery, tissue distribution, and metabolic effects of conjugated linoleic acid in rats. Journal of Nutrition Biochemistry 8, 38-43.

Szymczyk B, Pisulewski PM, Hanczakowski P \& Szczurek W (2000) The effects of feeding conjugated linoleic acid on rat growth performance, serum lipoproteins and subsequent lipid composition of selected rat tissues. Journal of the Science of Food and Agriculture 80, 1553-1558.

Thiel RL, Sparks JC, Weigand BR, Parrish FC \& Ewan RC (1998) Conjugated linoleic acid improves growth performance and body composition in swine. Journal of Animal Science 76, Suppl. 2, 61 (Abst.).

Visonneau S, Cesano A, Tepper SA, Scimeca JA, Santoli D \& Kritchevsky D (1997) Conjugated linoleic acid supresses the growth of human breast adenocarcinoma cells in SCID mice. Anticancer Research 17, 969-974.

Viviani R (1970) Metabolism of long-chain fatty acids in the rumen. Advances in Lipid Research 8, 267-274.

Warrick GR, Berdersond J \& Alberts JJ (1982) Dextran-sulphate$\mathrm{Mg}$ precipitation of high-density lipoprotein cholesterol. Clinical Chemistry 28, 1379-1388.

West DB, Delany JP, Camet PM, Blohm F, Truett AA \& Scimeca J (1998) Effects of conjugated linoleic acid on body fat and energy metabolism in the mouse. American Journal of Physiology 44, R667-R672.

Wong MW, Chew BP, Wong TS, Hosick HL, Boylston TD \& Schulz TD (1998) Effects of dietary conjugated linoleic acid on lymphocyte function and growth of mammary tumors in mice. Anticancer Research 17, 987993. 